\title{
Tuning the Rectification Ratio by Changing the Electronic Nature (Open-Shell and Closed-Shell) in Donor-Acceptor SAMs
}

\author{
Manuel Souto, ${ }^{\dagger}$ Li Yuan, ${ }^{\ddagger}$ Dayana C. Morales, ${ }^{\dagger}$ Li Jiang, ${ }^{\ddagger}$ Imma Ratera, ${ }^{\dagger}$ Christian A. Nijhuis, ${ }^{\ddagger, \Phi},{ }^{*}$ \\ Jaume Veciana ${ }^{\dagger, *}$ \\ ${ }^{\dagger}$ Institut de Ciència de Materials de Barcelona (ICMAB-CSIC)/CIBER-BBN, Campus de la UAB, o8193 Bellaterra, \\ Spain \\ ${ }^{\ddagger}$ Department of Chemistry, National University of Singapore, 3 Science Drive 3, Singapore 117543, Singapore \\ 'Centre for Advanced 2D Materials and Graphene Research Centre, National University of Singapore, 6 Science Drive
}

2, Singapore 117546, Singapore.

Supporting Information Placeholder

\begin{abstract}
This article describes the mechanism of charge transport across self-assembled monolayers (SAMs) of two donor-acceptor (D-A) systems consisting of polycholotriphenylmethyl (PTM) electron-acceptor moiety linked to an electron-donor ferrocene $(\mathrm{Fc})$ unit supported by ultraflat template-stripped $\mathrm{Au}\left(\mathrm{Au}^{\mathrm{TS}}\right)$ and contacted by eutec-tic alloy of gallium and indium (EGaIn) top contacts. The electronic and supramolecular structures of these SAMs were well-characterized. The PTM unit can be switched between the non-radical (1) and radical (2) forms which influences the rectification behavior of the junction. Junctions with non-radical units (1) rectify currents via the highest occupied molecular orbital (HOMO) with a rectification ratio $\mathrm{R}$ of 99, but junctions with radical units (2) have a new accessible state, a single-unoccupied molecular orbital (SUMO), which turns rectification off and $\mathrm{R}$ drops to 6.
\end{abstract}

One of the main challenges in molecular electronics is to relate charge transport through a single molecule, or a self-assembled monolayer (SAM), to the chemical and electronic structure of the molecular component of the molecular electronic device. ${ }^{1}$ In practice, this goal is not always straightforward to achieve because supramolecular and electronic structure of molecular junctions are difficult to control and characterize. ${ }^{2-5}$ Dyads formed by a donor unit (D) covalently linked to an acceptor (A) unit by an organic bridge are very promising materials for applications as molecular rectifiers. Indeed, the first molecular rectifier, proposed by Aviram and Ratner in 1974, was based on a D- $\sigma$-A dyad. ${ }^{6}$ Since this first theoretical proposal, several examples of D-A dyads have been studied as molecular rectifiers. ${ }^{7}$ In general, molecular diodes with rectification ratios $R>10$ are difficult to form and only a few examples have been reported with high values of $R$ of $10^{2}-10^{3},{ }^{8-10}$ because it is difficult to control the energy level alignment and the supramolecular structure, to minimize defects, and to reduce leakage currents. ${ }^{5}$

During the last years, some of us have studied in detail the rectifying properties of several SAMs based on alkanethiolates with ferrocene $(\mathrm{Fc}$ ) head groups concluding that the presence of energetically accessible molecular orbitals (the HOMO of the $\mathrm{Fc}$ ) is necessary to obtain large values of $R$ up to $1.1 \times 10^{3} .^{11-13}$ The mechanism of these diodes have been confirmed by others experimentally, ${ }^{14-16}$ and they works as follow. The HOMO of the molecules centered at the Fc units is slightly lower in energy than the Fermi levels of the electrodes. At positive bias, the HOMO cannot participate in charge transport and the Fc moieties are part of the tunneling barrier and the mechanism of charge transport is dominated by temperature independent coherent tunneling. In contrast, at negative bias the HOMO can participate in the mechanism of charge transport and temperature dependent incoherent tunneling occurs (in other words, Fc is acting as a hopping center). The performance of the molecular diodes also strongly depends on the degree of asymmetry of the molecular component measured by the difference in the coupling parameter $\Gamma_{\mathrm{i}}(\mathrm{i}$ indicates top (t) or bottom (b) electrode) of the molecular orbitals (MOs) with the Fermi level of the electrodes. The value of $\Gamma$ is related to the potential drop over the junctions, and the asymmetry is parameterised with the dimensionless division parameter $\eta_{V}=\Gamma_{\mathrm{i}} /\left(\Gamma_{\mathrm{b}}+\Gamma_{\mathrm{t}}\right)$ and junction rectify when $\eta_{\mathrm{V}}$ is close to o or 1 . The mechanism of the rectification has been recently described in detail. ${ }^{5,17}$ On the other hand, we have also recently reported the charge transport properties of SAMs based on alkanethiolates with another electroactive head group, a polychlorotriphenylmethyl (PTM) radical placed close to the top-electrode, which, surprisingly, did not rectify. ${ }^{18}$ Here we wish to investigate in detail the mechanisms of charge transport whether it is possible to turn rectification on and off by combining the Fc and PTM units within a single SAM.

Some of us have reported D-A dyads based on the Fc electron-donor linked to the PTM electron-acceptor via a $\pi$-conjugated vinylene bridge. These D-A systems exhibited bistability in a neutral and a zwitterionic states. ${ }^{19,20}$ Here, we designed and synthesized two new Fc-PTM dyads incorporating a terminal disulfide (DS) group to form SAMs on gold surfaces. We incorporated 
these SAMs in EGaIn junctions (Figure 1) to study the mechanism of charge transport with the PTM units in their non-radical (1, DS-Fc-PTM-NR) and radical (2, DS-Fc-PTM-R) forms (Figure 1). Interestingly, junctions with 1 are good molecular diodes with $R$ $=99$ but $R$ decreased to 6 for the same junction but with 2 . We attribute this decrease in $R$ to the electronic structure of the SAMs since both SAMs have the same supramolecular structure and we only changed the PTM units between their closed- and open-shell structures. In addition, we also synthesized and prepared SAMs of a Fc functionalized with a cyclic disulfide (3, DSFc), lacking the PTM moiety, that we used as a reference. This system helped us to understand the differences in the current densities $J$ (in $\mathrm{A} / \mathrm{cm}^{2}$ ) as well as in the charge transport mechanism of $\mathbf{1}$ and $\mathbf{2}$.
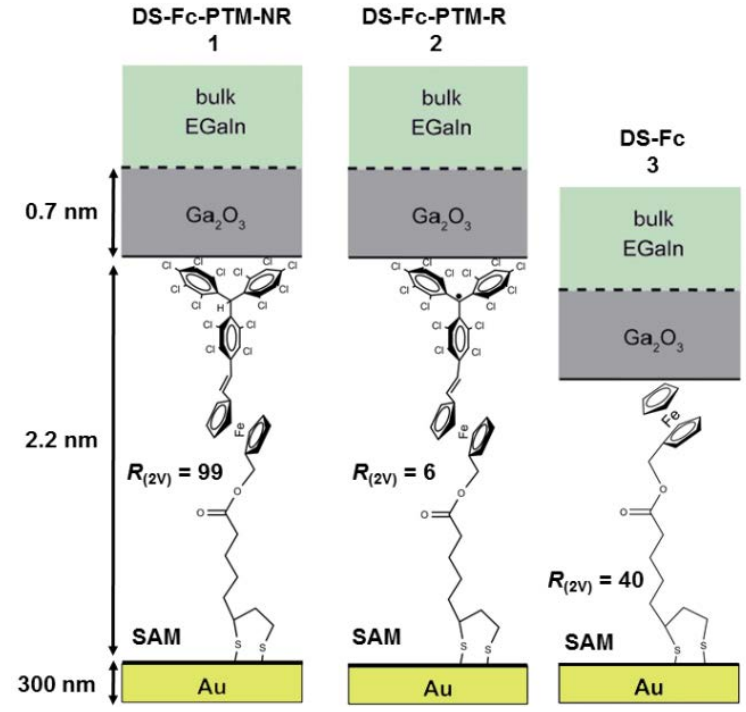

Figure 1. Idealized schematic representations of the junctions based on Au bottom electrodes, SAMs 1, 2, and 3, and $\mathrm{Ga}_{2} \mathrm{O}_{3} /$ EGaIn top electrodes.

We synthesized compounds 1, 2, and 3, as reported in the Supporting Information (Scheme S1). SAMs 1, 2 and 3 were prepared following a previously reported procedure ${ }^{18}$ by immersion of template-stripped $\mathrm{Au}\left(\mathrm{Au}^{\mathrm{TS}}\right)$ substrates in $\mathbf{1 . 0} \mathrm{mM}$ solution of $\mathbf{1}$, $\mathbf{2}$, and 3 , in toluene under a nitrogen atmosphere. The functionalized gold substrates were rinsed thoroughly with anhydrous toluene to remove any physisorbed material. We characterized the SAMs by means of Time-of-flight secondary ion mass spectrometry (ToF-SIMS), electron spin resonance (ESR), cyclic voltammetry (CV), PM-IRRAS, photoemission spectroscopy (PES), and near edge X-ray absorption fine structure (NEXAFS) spectroscopy (see Sup. Inf.). Figure Siz shows the cyclic voltammograms of the SAMs derived from 1 and 2, on Au measured at scan rates $(v)$ of o.1-1.o V/s. The reversible redox process assigned to the oxidation of the Fc unit was observed at $E_{1 / 2}{ }^{o x}=0.70$ and $0.67 \mathrm{~V}$ for SAM 1 and 2, respectively. The CV of SAM 2 also exhibited the reduction wave of the PTM radical moiety at $E_{1 / 2}^{\text {red }}=-0.14 \mathrm{~V}$. The insets confirm that the redox couples were immobilized as evidenced by the linear correlation between $i_{\mathrm{pc}}$ and $v$. The value of $\Delta E_{\mathrm{p}}\left(=E_{\mathrm{pa}}-E_{\mathrm{pc}}\right)$ was in the range of 20-35 $\mathrm{mV}$ as expected for a surface confined redox couple. Integration of the oxidation waves gave the total charge from which determined the surface coverage of $1-2 \times 10^{-10} \mathrm{~mol} / \mathrm{cm}^{2}$ (Table 1 ).

The electronic structures of the SAMs were characterized with ultraviolet photoemission spectroscopy (UPS) and near-edge Xray absorption (NEXAFS) of the C K-edge. The UPS data of SAM 1 and 2 showed a peak at 0.75 and o.65 eV, respectively, assigned to the HOMO localized on the Fc units appearing at similar energies for both SAMs (Figure S18). We recorded NEXAFS spectra of both SAMs at normal and grazing incidence (Figure S19). The main difference between both NEXAFS spectra was the small peak at $282.6 \mathrm{eV}$ in SAM 2 assigned to the single unoccupied molecular orbital (SUMO) localized on the PTM radical. ${ }^{18,21}$ The lowest unoccupied molecular orbitals (LUMO) were also determined. Thus, we determined the HOMO, LUMO, and SUMO energy levels (Table 1) of the closed- and open-shell SAM 1 and $\mathbf{2}$ which have largely one difference: the absence (SAM $\mathbf{1}$ ) or presence (SAM 2) of the SUMO while retaining the same supramolecular structure. The SOMO orbital was estimated to be at lower energies than the HOMO $(-5.7 \mathrm{eV})$ as it has been reported for other PTM-based SAMs derivatives. ${ }^{17}$ The orbital energy levels were used to construct the energy level diagrams shown in Figure 3 below. The UPS spectra show that the work function of the Au electrodes shift to $4.2 \mathrm{eV}$ as described in our earlier works, and that by others. ${ }^{17,22,23}$ It is well-known that these shifts in work functions are caused by push-back effect as result of the Au-S bond. 

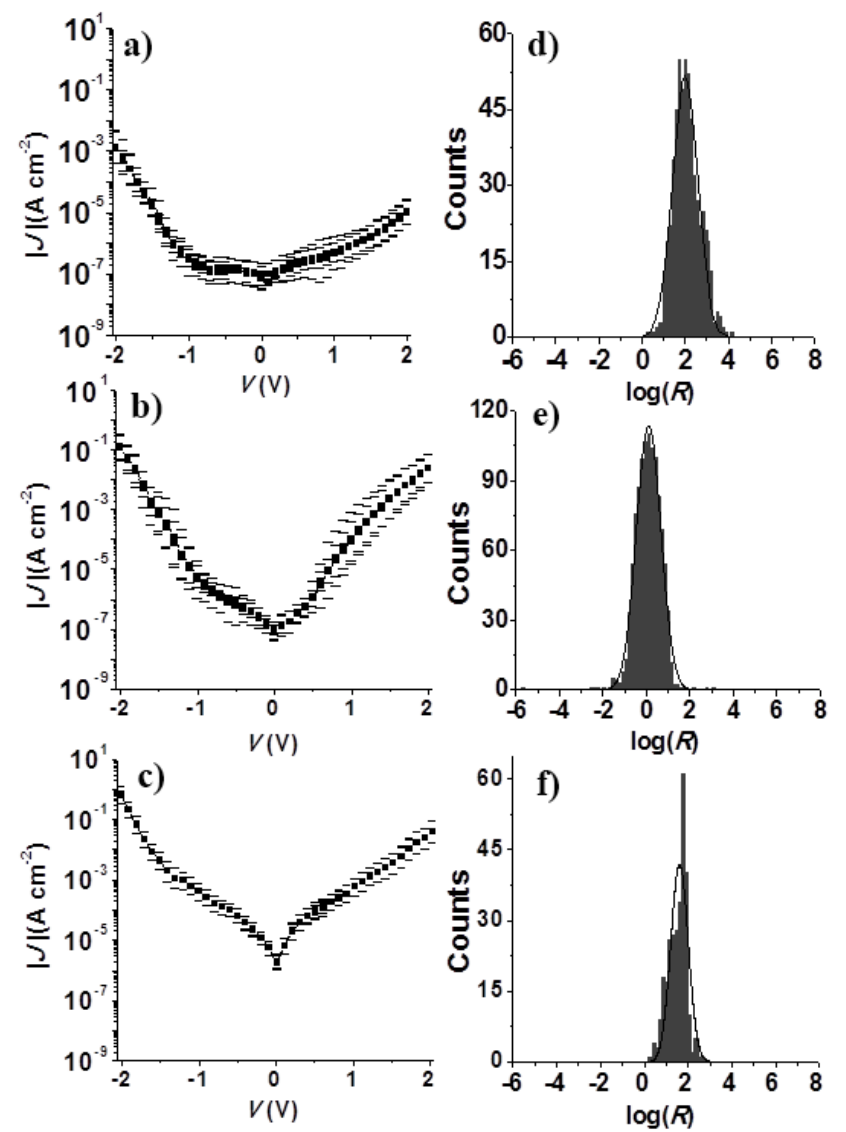

Figure 2. The $|J|(\mathrm{V})$ of the junctions formed by a) $\mathrm{Au}^{\mathrm{TS}}-\mathrm{SAM1} / / \mathrm{Ga}_{2} \mathrm{O}_{3} / \mathrm{EGaIn}$, b) $\mathrm{Au}^{\mathrm{TS}}-\mathrm{SAM} 2 / / \mathrm{Ga}_{2} \mathrm{O}_{3} / \mathrm{EGaIn}$ and c) $\mathrm{Au}^{\mathrm{TS}}$ $\mathrm{SAM}_{3} / / \mathrm{Ga}_{2} \mathrm{O}_{3} /$ EGaIn along with histograms (d-f), with Gaussian fits to these histograms, of $R$ determined at $\pm 2.0 \mathrm{~V}$.

a)
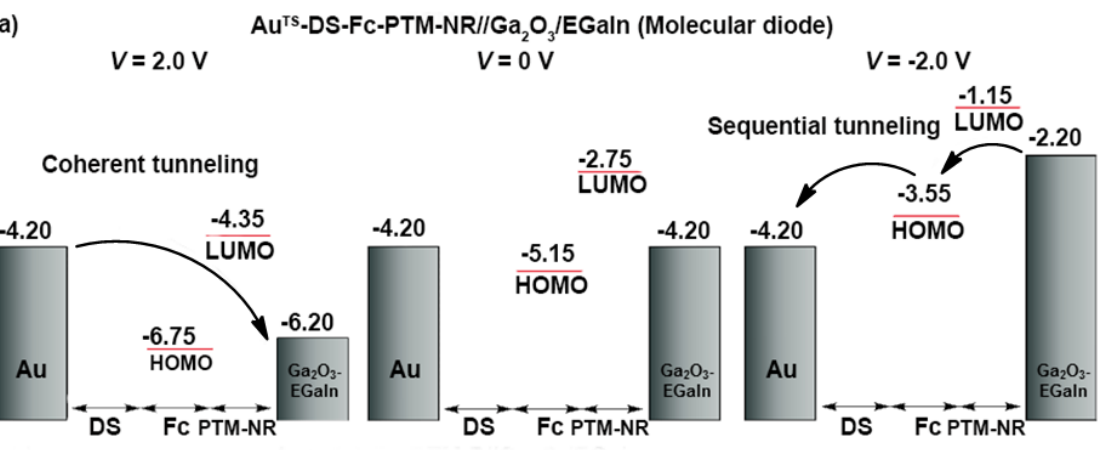

b)

$\mathrm{Au}^{\mathrm{TS}}$-DS-FC-PTM-NR//Ga $\mathrm{O}_{3} /$ EGaln (Molecular non-diode)

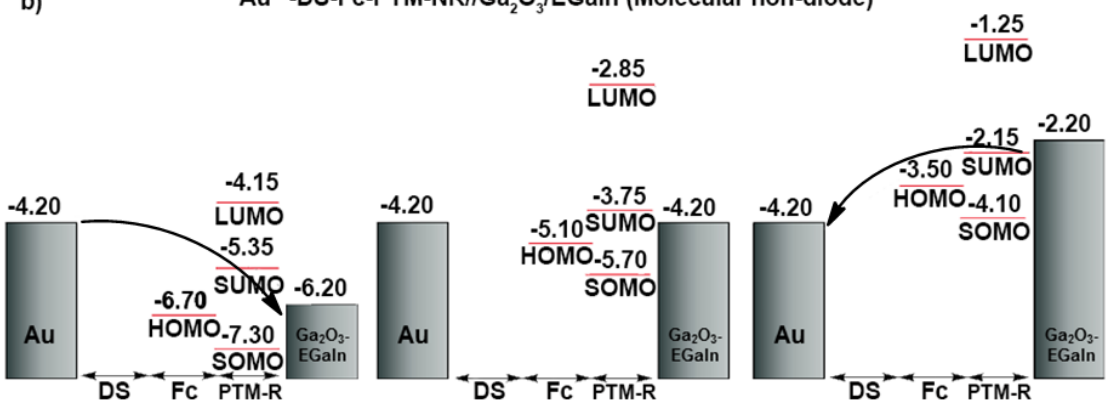

Figure 3. Energy level diagrams (with respect to the vacuum) of the a) $\mathrm{Au}^{\mathrm{TS}}-\mathrm{SAM1} / / \mathrm{Ga}_{2} \mathrm{O}_{3} / \mathrm{EGaIn}$, b) $\mathrm{Au}^{\mathrm{TS}}-$ SAM2// $\mathrm{Ga}_{2} \mathrm{O}_{3} /$ EGaIn junctions at 2.0 V (left), o V (middle), and -2.o V (right) based on the experimental data in Table 1. We biased the $\mathrm{Ga}_{2} \mathrm{O}_{3} /$ EGaIn top electrode and grounded the $\mathrm{Au}^{\mathrm{TS}}$ bottom electrode. DS stands for disulfide alkyl chain. 
The arrows indicate the orbitals that are involved in the electron transfer process. The mechanism of the rectification of the diode is indicated in the figure and detailed in Refs. ${ }^{5,17}$.

Table 1. Physico-chemical properties of SAMs 1 and 2.

\begin{tabular}{|c|c|c|c|c|c|c|}
\hline & \multicolumn{2}{|c|}{ UPS } & \multicolumn{2}{|c|}{ NEXAFS } & \multicolumn{2}{|c|}{ XPS } \\
\hline $\begin{array}{l}\text { SA } \\
\text { M }\end{array}$ & $\begin{array}{l}\text { WF } \\
(\mathrm{eV})\end{array}$ & $\begin{array}{c}\boldsymbol{E}_{\text {Номо }} \\
(\mathrm{eV})^{\mathrm{a}}\end{array}$ & $\begin{array}{c}\text { SUMO } \\
(\mathrm{eV})\end{array}$ & $\begin{array}{c}\text { LUMO } \\
(\mathrm{eV})\end{array}$ & $\begin{array}{l}\text { (n } \\
\mathbf{m} \\
)^{b}\end{array}$ & $\begin{array}{c}\Gamma_{\mathrm{SAM}} \\
\left(\times \mathbf{1 0}^{-10}\right. \\
\mathrm{mol} / \mathrm{c} \\
\left.\mathrm{m}^{2}\right)^{\mathrm{c}}\end{array}$ \\
\hline 1 & 4.40 & -5.15 & N.A. & -2.75 & 2.2 & 1.50 \\
\hline 2 & 4.45 & -5.10 & -3.75 & -2.85 & 2.3 & 1.50 \\
\hline
\end{tabular}

${ }^{a} \mathrm{HOMO}$ energies calculated from UPS. The error is $0.05 \mathrm{eV}$, which is the resolution of the UPS. ${ }^{b}$ Thickness of SAMs calculated from XPS data. ${ }^{c}$ Surface coverage calculated from XPS data.

Figure 2 shows that junctions based on SAM 2 have at all assayed bias $\sim 100$ times higher values of $J$ than those junctions with SAM 1 in agreement with previous findings. ${ }^{18,24}$ Even though we can still observe a kink in the shape of $J(\mathrm{~V})$ curve at $\sim-1.0$ V similar to junctions with SAM 1, which indicates that the HOMO enters the conduction window, the current at positive bias (around $+0.3 \mathrm{~V}$ ) increases sharply indicating that the SUMO enters the bias window diminishing the rectification ratio. In a recent study we showed that the SUMO of a PTM unit can participate in the mechanism of charge transport and increases the tunneling rate. ${ }^{18}$ Here we believe a similar mechanism of charge transport dominates and Figure 3 shows that the SUMO of SAM 2 provides a new tunneling pathway inside the HOMO-LUMO gap which explains the 10o-fold increase in $J$.

Interestingly, the junctions with SAM 1 rectify with a value of $R=|J(-2.0 \mathrm{~V})| / \mid J(+2 . \mathrm{oV})$ of nearly two orders of magnitude $(\sim 99)$ whereas junctions with SAM 2 marginally rectified current $(\sim 6)$. The energy level diagrams shown in Figure 3 were constructed using the work functions and the HOMO onset values from the UPS data, and the LUMO/SUMO levels estimated by NEXAFS (Table 1). We believe that the mechanism of rectification is similar to that observed for junctions with a single Fc unit located asymmetrically inside the junction. The Fc, and Fc-PTM units, are electronically decoupled from the bottom-electrode due to the large potential drop across the insulating alkyl chain, but they are coupled to the top-electrode via van der Waals interactions. These van der Waals interactions are strong enough for the frontier orbitals to track the changes in the Fermi-level of the top-electrode when the junctions are biased. ${ }^{5,17}$ Due to the aromatic nature of the PTM unit, we assumed that the potential drop along this part of the molecule will be much smaller than across the insulating aliphatic linker. In other words, the potential drop across the $\mathrm{Fc} /$ /top electrode interface is small and here we assume that $0.4 \mathrm{~V}$ of the $2 \mathrm{~V}$ applied potential drops due to the PTM unit. Figure za shows that this mechanism involves tunneling via the HOMO that only falls in the conductions window at negative bias and not at positive bias. To test this hypothesis, we also made junctions with SAM 3 (i.e., the junctions but without the PTM unit) and indeed these junctions also rectify with $R=40$. The values of $J$ for junctions with SAM 3 are a factor of $3.16 \times 10^{2}$ higher than those of junctions with SAM 1. This result agree with our previous findings were the PTM moiety has a large HOMO-LUMO gap and thus adds to width of the tunneling barrier and lowers $J$. The fact that junctions with SAM $\mathbf{1}$ rectify, despite the fact that the Fc units are separated from the top electrode by the PTM moiety lowering $\eta$ (the Fc units are approximately located in the middle of the junction), can be explained by a large intramolecular coupling between the Fc and PTM so that the Fc unit still can follow the changes to the Fermi-level of the top electrode. In other words, we believe that due to the conjugated nature of the PTM units that the potential drop across them is relatively flat, as indicated in the Figure 3 , so that the HOMO (centered at the Fc unit) can still follow the changes of the Fermi level during applied bias (i.e., the Fc units are capacitively stronger coupled to the top- than the bottom-electrode) and can participate in the mechanism of charge transport only at negative bias. On the other hand, junctions with SAM 2 do not rectify. Here, the SUMO is close in energy to the Fermi levels of the electrodes and falls in the conduction window at both bias polarities (Fig. 3b). As mentioned above, the SUMO provides an inter HOMO-LUMO gap tunneling pathway resulting in high value of $J$ and the junctions stop to rectify despite their asymmetry. Although we infer here a coherent transport pathway mediated by the PTM unit based on previous works, additional experiments are needed to investigate the mechanism in more detail.

In summary, we have reported two D-A systems (1 and 2) consisting on the electron-acceptor PTM, with closed- and openshell electronic structure, linked to the electron donor Fc moiety that were immobilized on a gold surface through a disulfide group. Interestingly, the non-radical SAM 1, which has a similar supramolecular structure but a different electronic one than SAM 2, showed a rectification behavior which was two orders of magnitude larger than its radical analogue. This different behavior was attributed to the participation of the SUMO orbital on the charge transport mechanism in SAM 2. Our designed D-A system provide the approach to control the potential drop over the whole junctions to achieve high rectification. Our results demonstrate that it is possible to control the rectification ratio by changing the electronic nature, i.e., closed- and open-shell structure, of the electron-acceptor unit of molecular dyads. Here we switched between the open and closed form by chemical means, and we believe that our findings are important towards the rational design of active molecular rectifiers.

\section{ASSOCIATED CONTENT}

\section{Supporting Information}

General methods for synthesis and characterization. Synthesis and characterization of 1, 2, and 3. General procedures for the SAMs preparation and characterization, and transport measurements.

\section{AUTHOR INFORMATION}

\section{Corresponding Author}


*chmnca@nus.edu.sg

*vecianaj@icmab.es

Notes

The authors declare no competing financial interests.

\section{ACKNOWLEDGMENT}

This work was supported by the EU ITN iSwitch 642196 DGI grant (BeWell; CTQ2013-4048o-R), the Networking Research Center on Bioengineering, Biomaterials, and Nanomedicine (CIBER-BBN), and the Generalitat de Catalunya (grant 2014-SGR-17). ICMAB acknowledges support from the Spanish Ministry of Economy and Competitiveness, through the "Severo Ochoa" Programme for Centres of Excellence in R\&D (SEV- 2015-0496). Prime Minister's Office, Singapore under its Medium sized centre program is also acknowledged for supporting this research. Yu Xiaojiang is kindly acknowledged for assisting at the SINS beam line at SSLS under NUS core support C-380-003-003-001. We also acknowledge the Ministry of Education (MOE) for supporting this research under award No. MOE2015-T2-1-050.

\section{REFERENCES}

(1) Metzger, R. M. Chem. Rev. 2015, 115, 5056-5115.

(2) McCreery, R. L. Anal. Chem. 2006, 78 (11), 3490-3497.

(3) McCreery, R. L. Chem. Mater. 2004, 16 (23), 4477-4496.

(4) Huang, C.; Rudnev, A. V; Hong, W.; Wandlowski, T. Chem. Soc. Rev. 2015, 44, 889-901.

(5) Thompson, D.; Nijhuis, C. A. Acc. Chem. Res. 2016, 49, 2061-2069.

(6) Aviram, A.; Ratner, M. A. Chem. Phys. Lett. 1974, 29 (2), 277-283.

(7) Metzger, R. M. Chem. Rev. 2003, 103 (9), 3803-3834.

(8) Capozzi, B.; Xia, J.; Adak, O.; Dell, E. J.; Liu, Z.-F.; Taylor, J. C.; Neaton, J. B.; Campos, L. M.; Venkataraman, L. Nat. Nanotechnol. 2015, 10 (6), 522-527.

(9) Perrin, M. L.; Galán, E.; Eelkema, R.; Thijssen, J. M.; Grozema, F.; van der Zant, H. S. J. Nanoscale 2016, 8 (16), 8919-8923.

(10) Yuan, L.; Breuer, R.; Jiang, L.; Schmittel, M.; Nijhuis, C. A. Nano Lett. 2015, 15 (8), 5506-5512.

(11) Nijhuis, C. a.; Reus, W. F.; Whitesides, G. M. J. Am. Chem. Soc. 2009, 131 (49), 17814-17827.

(12) Nijhuis, C. a.; Reus, W. F.; Whitesides, G. M. J. Am. Chem. Soc. 2010, 132 (51), 18386-18401.

(13) Nerngchamnong, N.; Yuan, L.; Qi, D.-C.; Li, J.; Thompson, D.; Nijhuis, C. a. Nat. Nanotechnol. 2013,8 (2), $113-118$.

(14) Nijhuis, C. a.; Reus, W. F.; Barber, J. R.; Dickey, M. D.; Whitesides, G. M. Nano Lett. 2010, 10 (9), $3611-3619$.

(15) Jeong, H.; Kim, D.; Wang, G.; Park, S.; Lee, H.; Cho, K.; Hwang, W. T.; Yoon, M. H.; Jang, Y. H.; Song, H.; Xiang, D.; Lee, T. Adv. Funct. Mater. 2014, 24 (17), 2472-2480.

(16) Mentovich, E. D.; Rosenberg-shraga, N.; Kalifa, I.; Gozin, M.; Mujica, V.; Hansen, T.; Richter, S. J. Phys. Chem. C 2013, 117, 84688474 .

(17) Yuan, L.; Nerngchamnong, N.; Cao, L.; Hamoudi, H.; del Barco, E.; Roemer, M.; Sriramula, R. K.; Thompson, D.; Nijhuis, C. a. Nat. Commun. 2015, 6, 6324.

(18) Yuan, L.; Franco, C.; Crivillers, N.; Mas-Torrent, M.; Cao, L.; Sangeeth, C. S. S.; Rovira, C.; Veciana, J.; Nijhuis, C. A. Nat. Commun. 2016, 7 (8), 12066.

(19) Ratera, I.; Ruiz-Molina, D.; Renz, F.; Ensling, J.; Wurst, K.; Rovira, C.; Gütlich, P.; Veciana, J. J. Am. Chem. Soc. 2oo3, 125, 14621463.

(20) Guasch, J.; Grisanti, L.; Jung, S.; Morales, D.; D’Avino, G.; Souto, M.; Fontrodona, X.; Painelli, A.; Renz, F.; Ratera, I.; Veciana, J. Chem. Mater. 2013, 25, 808-814.

(21) Mugnaini, V.; Calzolari, A.; Ovsyannikov, R.; Vollmer, A.; Gonidec, M.; Alcon, I.; Veciana, J.; Pedio, M. J. Phys. Chem. Lett. 2o15, 2101-2106.

(22) Boer, B. De; Hadipour, A.; Mandoc, M. M.; Woudenbergh, T. Van; Blom, P. W. M. Adv. Mater. 2005, No. 5, $621-625$.

(23) Cahen, D.; Kahn, A.; Umbach, E. Mater. Today 2005, 8 (7), 32-41.

(24) Crivillers, N.; Munuera, C.; Mas-Torrent, M.; Simão, C.; Bromley, S. T.; Ocal, C.; Rovira, C.; Veciana, J. Adv. Mater. 20o9, 21 (1011), 1177-1181. 

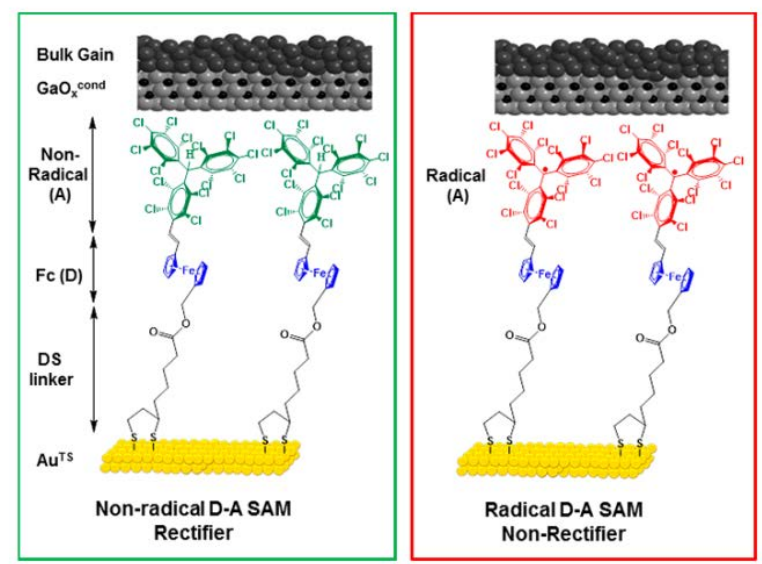